\title{
ON THE LAGRANGE RESOLVENTS OF A DIHEDRAL QUINTIC POLYNOMIAL
}

\author{
BLAIR K. SPEARMAN and KENNETH S. WILLIAMS
}

Received 14 July 2004

\begin{abstract}
The cyclic quartic field generated by the fifth powers of the Lagrange resolvents of a dihedral quintic polynomial $f(x)$ is explicitly determined in terms of a generator for the quadratic subfield of the splitting field of $f(x)$.
\end{abstract}

2000 Mathematics Subject Classification: 11R16, 11R21.

Let $f(x)=x^{5}+p x^{3}+q x^{2}+r x+s \in \mathbb{Q}[x]$ be an irreducible quintic polynomial with a solvable Galois group. Let $x_{1}, x_{2}, x_{3}, x_{4}, x_{5} \in \mathbb{C}$ be the roots of $f(x)$. The splitting field of $f$ is $K=\mathbb{Q}\left(x_{1}, x_{2}, x_{3}, x_{4}, x_{5}\right)$. Let $\zeta$ be a primitive fifth root of unity. The Lagrange resolvents of the root $x_{1}$ are

$$
\begin{aligned}
& r_{1}=\left(x_{1}, \zeta\right)=x_{1}+x_{2} \zeta+x_{3} \zeta^{2}+x_{4} \zeta^{3}+x_{5} \zeta^{4} \in K(\zeta), \\
& r_{2}=\left(x_{1}, \zeta^{2}\right)=x_{1}+x_{2} \zeta^{2}+x_{3} \zeta^{4}+x_{4} \zeta+x_{5} \zeta^{3} \in K(\zeta), \\
& r_{3}=\left(x_{1}, \zeta^{3}\right)=x_{1}+x_{2} \zeta^{3}+x_{3} \zeta+x_{4} \zeta^{4}+x_{5} \zeta^{2} \in K(\zeta), \\
& r_{4}=\left(x_{1}, \zeta^{4}\right)=x_{1}+x_{2} \zeta^{4}+x_{3} \zeta^{3}+x_{4} \zeta^{2}+x_{5} \zeta \in K(\zeta) .
\end{aligned}
$$

We set

$$
R_{i}=r_{i}^{5}, \quad i=1,2,3,4
$$

By [1, Theorem 2] we know that the Galois group of $f$ is $\mathbb{Z}_{5}$ (cyclic group of order 5), $D_{5}$ (dihedral group of order 10), or $F_{20}$ (Frobenius group of order 20). When $\operatorname{Gal}(f) \simeq D_{5}$, the splitting field $K$ of $f$ contains a unique quadratic subfield, say $\mathbb{Q}(\sqrt{m})$ ( $m$ squarefree integer $\neq 1$ ). In this note we show, for quintic polynomials $f$ with $\operatorname{Gal}(f) \simeq D_{5}$, that the fields $\mathbb{Q}\left(R_{i}\right)(i=1,2,3,4)$ are the same cyclic quartic field and we give a simple explicit generator for this field. We prove the following theorem.

THEOREM 1. If $\mathrm{Gal}(f) \simeq D_{5}$, then

$$
\mathbb{Q}\left(R_{i}\right)=\mathbb{Q}(\sqrt{-m(5+2 \sqrt{5})}), \quad i=1,2,3,4,
$$

where $\mathbb{Q}(\sqrt{m})$ is the unique quadratic subfield of the splitting field $K$ of $f$. 
Proof. Expanding $\left(x_{1}, \zeta\right)^{5}=\left(x_{1}+x_{2} \zeta+x_{3} \zeta^{2}+x_{4} \zeta^{3}+x_{5} \zeta^{4}\right)^{5}$ we obtain

$$
R_{1}=l_{0}+l_{1} \zeta+l_{2} \zeta^{2}+l_{3} \zeta^{3}+l_{4} \zeta^{4}
$$

where $l_{0}, l_{1}, l_{2}, l_{3}, l_{4} \in K$ are given in [1, page 391$]$ and satisfy

$$
l_{0}+l_{1}+l_{2}+l_{3}+l_{4}=\left(x_{1}+x_{2}+x_{3}+x_{4}+x_{5}\right)^{5}=0 .
$$

As $\operatorname{Gal}(f) \simeq D_{5}$, by [1, Theorem 2, page 397] the discriminant $D$ of $f$ is a square in $\mathbb{Q}$. Thus, by [1, pages 392-397], $l_{1}, l_{2}, l_{3}, l_{4}$ are the roots of a quartic polynomial belonging to $\mathbb{Q}[x]$, which factors over $\mathbb{Q}$ into two irreducible conjugate quadratics

$$
\left(x^{2}+\left(T_{1}+T_{2} \sqrt{D}\right) x+\left(T_{3}+T_{4} \sqrt{D}\right)\right)\left(x^{2}+\left(T_{1}-T_{2} \sqrt{D}\right) x+\left(T_{3}-T_{4} \sqrt{D}\right)\right)
$$

with $T_{1}, T_{2}, T_{3}, T_{4} \in \mathbb{Q}$. The roots of one of these quadratics (without loss of generality the first) are $l_{1}$ and $l_{4}$, and the roots of the other are $l_{2}$ and $l_{3}$. Thus

$$
\begin{array}{cc}
l_{1}+l_{4}=-T_{1}-T_{2} \sqrt{D}, & l_{2}+l_{3}=-T_{1}+T_{2} \sqrt{D}, \\
l_{1} l_{4}=T_{3}+T_{4} \sqrt{D}, & l_{2} l_{3}=T_{3}-T_{4} \sqrt{D} .
\end{array}
$$

Clearly $\left[\mathbb{Q}\left(l_{i}\right): \mathbb{Q}\right]=2(i=1,2,3,4)$. Also $l_{i} \in K(i=1,2,3,4)$ so that $\mathbb{Q}\left(l_{i}\right) \subseteq K(i=$ $1,2,3,4)$. However $K$ has a unique quadratic subfield $\mathbb{Q}(\sqrt{m})$. Thus $\mathbb{Q}\left(l_{i}\right)=\mathbb{Q}(\sqrt{m})$, $i=1,2,3,4$. Hence

$$
l_{1}=a+b \sqrt{m}, \quad l_{4}=a-b \sqrt{m}, \quad l_{2}=c+d \sqrt{m}, \quad l_{3}=c-d \sqrt{m},
$$

where $a, b, c, d \in \mathbb{Q}, b \neq 0$ and $d \neq 0$. Thus

$$
l_{0}=-l_{1}-l_{2}-l_{3}-l_{4}=-2 a-2 c .
$$

Next we define

$$
g(x)=\left(x-R_{1}\right)\left(x-R_{2}\right)\left(x-R_{3}\right)\left(x-R_{4}\right) \in K(\zeta)[x] .
$$

Hence, as $1+\zeta+\zeta^{2}+\zeta^{3}+\zeta^{4}=0$, we obtain

$$
\begin{aligned}
R_{1}= & l_{0}+l_{1} \zeta+l_{2} \zeta^{2}+l_{3} \zeta^{3}+l_{4} \zeta^{4} \\
= & (a+b \sqrt{m}+2 a+2 c) \zeta+(c+d \sqrt{m}+2 a+2 c) \zeta^{2} \\
& +(c-d \sqrt{m}+2 a+2 c) \zeta^{3}+(a-b \sqrt{m}+2 a+2 c) \zeta^{4} \in \mathbb{Q}(\sqrt{m}, \zeta) .
\end{aligned}
$$

Similarly

$$
\begin{aligned}
R_{2}= & (a+b \sqrt{m}+2 a+2 c) \zeta^{2}+(c+d \sqrt{m}+2 a+2 c) \zeta^{4} \\
& +(c-d \sqrt{m}+2 a+2 c) \zeta+(a-b \sqrt{m}+2 a+2 c) \zeta^{3} \in \mathbb{Q}(\sqrt{m}, \zeta), \\
R_{3}= & (a+b \sqrt{m}+2 a+2 c) \zeta^{3}+(c+d \sqrt{m}+2 a+2 c) \zeta \\
& +(c-d \sqrt{m}+2 a+2 c) \zeta^{4}+(a-b \sqrt{m}+2 a+2 c) \zeta^{2} \in \mathbb{Q}(\sqrt{m}, \zeta), \\
R_{4}= & (a+b \sqrt{m}+2 a+2 c) \zeta^{4}+(c+d \sqrt{m}+2 a+2 c) \zeta^{3} \\
& +(c-d \sqrt{m}+2 a+2 c) \zeta^{2}+(a-b \sqrt{m}+2 a+2 c) \zeta \in \mathbb{Q}(\sqrt{m}, \zeta) .
\end{aligned}
$$


Using Maple we find that

$$
\begin{aligned}
g(x)= & x^{4}+(10 c+10 a) x^{3}+\left(5 b^{2} m+5 d^{2} m+80 a c+35 a^{2}+35 c^{2}\right) x^{2} \\
+ & \left(30 c d^{2} m+50 c^{3}+200 a^{2} c-20 b c d m+30 a b^{2} m+20 a d^{2} m\right. \\
& \left.+20 b^{2} c m+200 a c^{2}+50 a^{3}+20 a b d m\right) x-10 b^{3} d m^{2}+150 a^{3} c \\
& +25 a^{2} d^{2} m+25 b^{2} c^{2} m-5 b^{2} d^{2} m^{2}+275 a^{2} c^{2}+25 c^{4}+10 b d^{3} m^{2} \\
+ & 50 a c d^{2} m-50 b c^{2} d m+150 a c^{3}+50 a^{2} b d m+50 c^{2} d^{2} m+5 d^{4} m^{2} \\
+ & 25 a^{4}+5 b^{4} m^{2}+50 a^{2} b^{2} m+50 a b^{2} c m .
\end{aligned}
$$

The roots of $g(x)$ are (again using Maple)

$$
\begin{aligned}
& -\frac{5}{2} a-\frac{5}{2} c+\frac{1}{2}(-a+c) \sqrt{5}+\frac{1}{2} \sqrt{-m\left(10\left(b^{2}+d^{2}\right)-\left(2 b^{2}+8 b d-2 d^{2}\right) \sqrt{5}\right)} \\
& -\frac{5}{2} a-\frac{5}{2} c+\frac{1}{2}(-a+c) \sqrt{5}-\frac{1}{2} \sqrt{-m\left(10\left(b^{2}+d^{2}\right)-\left(2 b^{2}+8 b d-2 d^{2}\right) \sqrt{5}\right)} \\
& -\frac{5}{2} a-\frac{5}{2} c-\frac{1}{2}(-a+c) \sqrt{5}+\frac{1}{2} \sqrt{-m\left(10\left(b^{2}+d^{2}\right)+\left(2 b^{2}+8 b d-2 d^{2}\right) \sqrt{5}\right)} \\
& -\frac{5}{2} a-\frac{5}{2} c-\frac{1}{2}(-a+c) \sqrt{5}+\frac{1}{2} \sqrt{-m\left(10\left(b^{2}+d^{2}\right)+\left(2 b^{2}+8 b d-2 d^{2}\right) \sqrt{5}\right)}
\end{aligned}
$$

The quantities under the radicals are $X+Y \sqrt{5}$ and $X-Y \sqrt{5}$, where

$$
X=-10 m\left(b^{2}+d^{2}\right), \quad Y=m\left(2 b^{2}+8 b d-2 d^{2}\right) .
$$

As

$$
X^{2}-5 Y^{2}=5 m^{2}\left(4 b^{2}-4 b d-4 d^{2}\right)^{2},
$$

the roots of $g(x)$ belong to the cyclic quartic field $\mathbb{Q}(\sqrt{X \pm Y \sqrt{5}})$ [2, Theorem 1 , page 134]. Further

$$
X+Y \sqrt{5}=(-10+2 \sqrt{5}) m\left(\frac{2 b-d-d \sqrt{5}}{2}\right)^{2}
$$

so that (as $b \neq 0$ and $d \neq 0$ )

$$
\mathbb{Q}(\sqrt{X+Y \sqrt{5}})=\mathbb{Q}(\sqrt{(-10+2 \sqrt{5}) m})=\mathbb{Q}(\sqrt{-m(5+2 \sqrt{5})}),
$$

as $(-10+2 \sqrt{5})(-5-2 \sqrt{5})=(5+\sqrt{5})^{2}$.

ACKNOWLEDGMENT. Both authors were supported by grants from the Natural Sciences and Engineering Research Council of Canada. 


\section{REFERENCES}

[1] D. S. Dummit, Solving solvable quintics, Math. Comp. 57 (1991), no. 195, 387-401.

[2] L.-C. Kappe and B. Warren, An elementary test for the Galois group of a quartic polynomial, Amer. Math. Monthly 96 (1989), no. 2, 133-137.

Blair K. Spearman: Department of Mathematics and Statistics, Okanagan University College, Kelowna, British Columbia, Canada V1V 1V7

E-mail address: bspearman@ouc.bc.ca

Kenneth S. Williams: School of Mathematics and Statistics, Carleton University, Ottawa, Ontario, Canada K1S 5B6

E-mail address: wi11 iams@math . car 1 eton.ca 


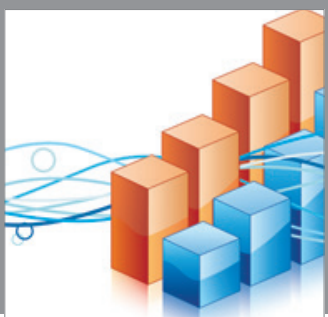

Advances in

Operations Research

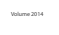

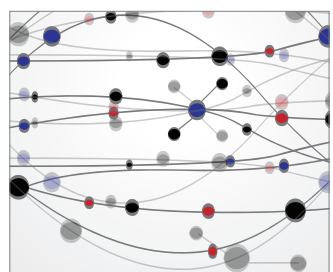

\section{The Scientific} World Journal
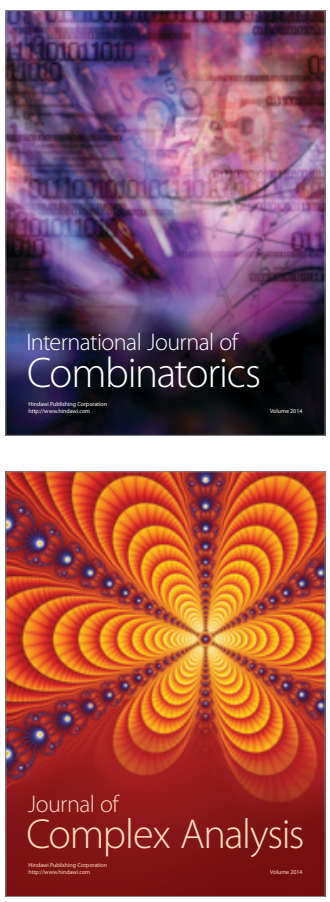

International Journal of

Mathematics and

Mathematical

Sciences
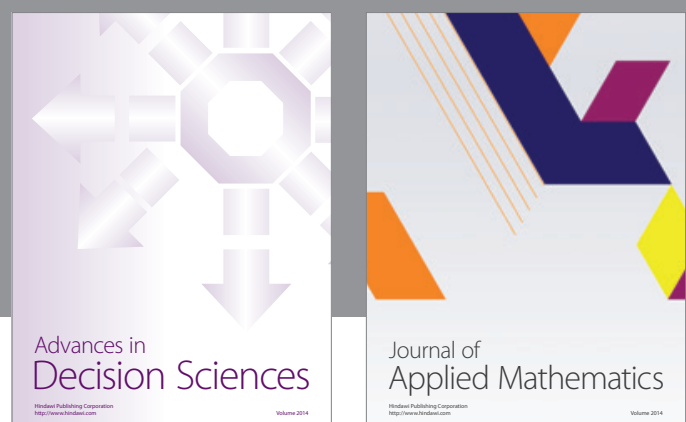

Journal of

Applied Mathematics
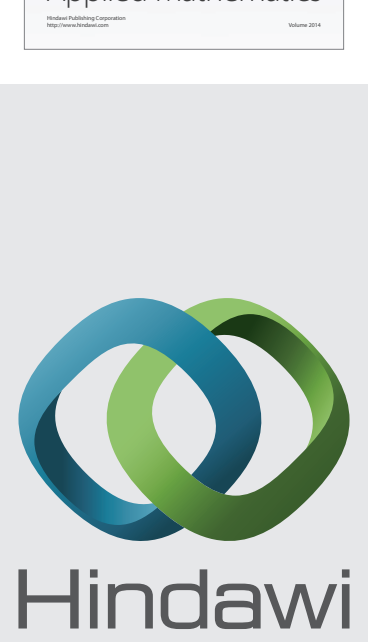

Submit your manuscripts at http://www.hindawi.com
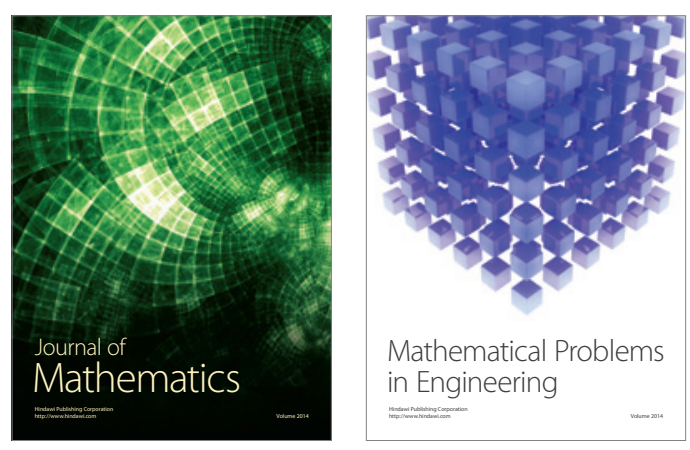

Mathematical Problems in Engineering
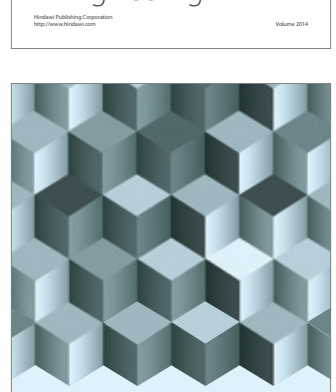

Journal of

Function Spaces
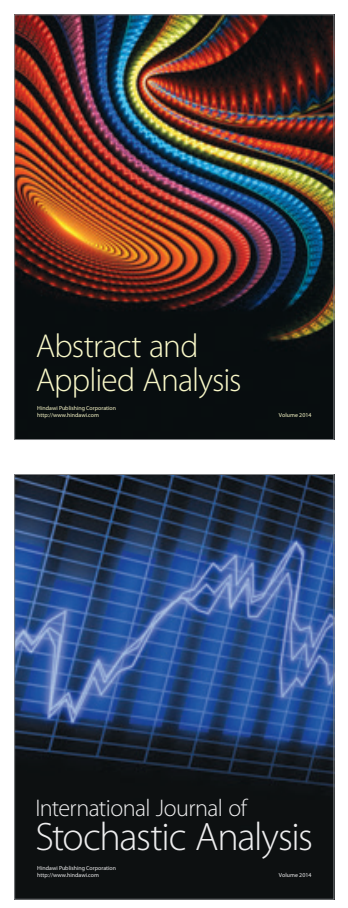

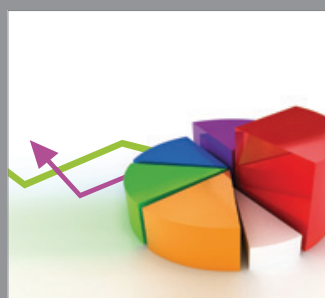

ournal of

Probability and Statistics

Promensencen
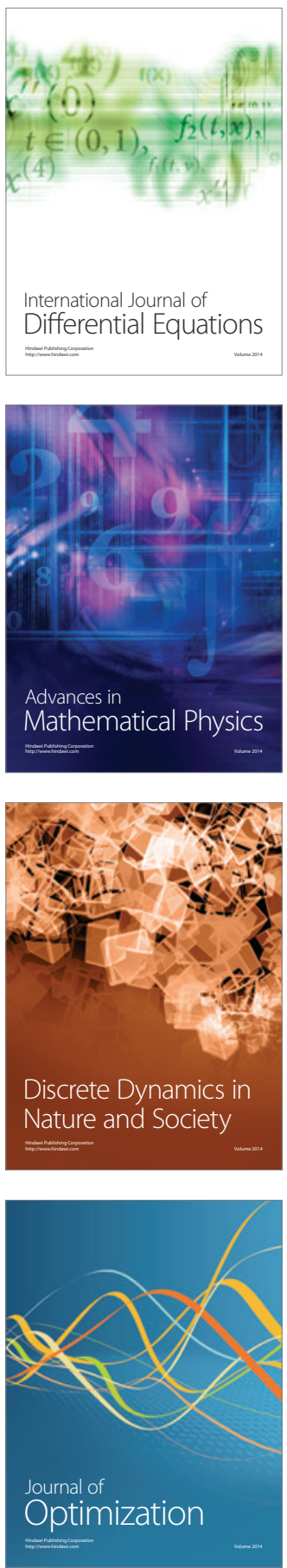